Trinity University

Digital Commons @ Trinity

Mathematics Faculty Research

Mathematics Department

$12-2006$

\title{
Asymptotic Sign-Solvability, Multiple Objective Linear Programming, and The Nonsubstitution Theorem
}

\section{Clayton}

R Herring

Allen G. Holder

Trinity University, aholder@trinity.edu

J Holzer

C Nightingale

See next page for additional authors

Follow this and additional works at: https://digitalcommons.trinity.edu/math_faculty

Part of the Mathematics Commons

\section{Repository Citation}

Cayton, L., Herring, R., Holder, A., Holzer, J., Nightingale, C., \& Stohs, T. (2006). Asymptotic sign-solvability, multiple objective linear programming, and the nonsubstitution theorem. Mathematical Methods of Operations Research, 64(3), 541-555. doi:10.1007/ s00186-006-0095-z

This Post-Print is brought to you for free and open access by the Mathematics Department at Digital Commons @ Trinity. It has been accepted for inclusion in Mathematics Faculty Research by an authorized administrator of Digital Commons @ Trinity. For more information, please contact jcostanz@trinity.edu. 
Authors

L Clayton, R Herring, Allen G. Holder, J Holzer, C Nightingale, and T Stohs 


\title{
Asymptotic Sign-Solvability, Multiple Objective Linear Programming, and The Nonsubstitution Theorem
}
L. Cayton ${ }^{a *}$
R. Herring ${ }^{b *}$
A. Holder ${ }^{c *}$
J. Holzer ${ }^{d *}$
C. Nightingale ${ }^{e *}$
T. Stohs ${ }^{f *}$

February 19, 2004

\begin{abstract}
In this paper we investigate the asymptotic stability of dynamic, multipleobjective linear programs. In particular, we show that a generalization of the optimal partition stabilizes for a large class of data functions. This result is based on a new theorem about asymptotic sign-solvable systems. The stability properties of the generalized optimal partition are used to extend a dynamic version of the Nonsubstitution Theorem.
\end{abstract}

Keywords: multiple-objective linear programming, asymptotic programming, sign-solvability, Nonsubstitution Theorem, computational economics

${ }^{a}$ Washington University, St. Louis Missouri, lac3@cec.wustl.edu

${ }^{b}$ Oberlin College, Oberlin Ohio, Ryan.Herring@oberlin.edu

${ }^{c}$ Trinity University Mathematics, San Antonio Texas, aholder@trinity.edu

${ }^{d}$ University of Wisconsin, Madison Wisconsin, holzer@math.wisc.edu

e Mills College, Oakland California, CNightin@mills.edu

${ }^{f}$ University of Nebraska-Lincoln, Lincoln Nebraska, tstohs1@bigred.unl.edu

* All research was conducted at Trinity University and was supported by NSF Grant 0097366. 


\section{Introduction \& Notation}

Linear programming is one of the most prominent areas in optimization, having a rich mathematical theory and numerous applications in economics, the management sciences, health care, and the physical sciences (to name just a few). In this paper, we focus on the connection between linear programming and economics. In particular, we extend the Nonsubstitution Theorem, which is an economics result that was first established by the Nobel Laureate P. Samuelson [21] and further studied by the Nobel Laureate J. Mirrlees [19]. This result states that under typical economic assumptions, there is an optimal manner to produce commodities that is independent of demand. The Nonsubstitution Theorem is considered by some to be the pivotal result that moved economics out of the neoclassical frame of thought and into the modern paradigm. The importance of this result is highlighted in the following quote [18], "The theorem was received with some astonishment by the authors working in the neoclassical tradition since it flatly contradicted the importance attached to consumer preferences for the determination of relative prices."

The Nonsubstitution Theorem continues to be investigated [7, 8, 10, 17], and in this paper we extend the main result of [13] to the case of multiple objectives. In [13], Hasfura-Buenaga, Holder, and Stuart studied a dynamic version of the Nonsubstitution Theorem and showed that the collection of optimal processes stabilizes, meaning that the collection is constant for sufficiently large time. One of the simplifying assumptions found in these works is that the only nonproducible commodity is a homogeneous labor source, and hence, every worker is paid the same wage. Our extension to the multiple-objective case removes this assumption and permits multiple labor sources with differing wages.

As in [13], our extension relies on the concept of the optimal partition. Consider the standard form primal and dual linear programs,

$$
(L P) \min \left\{c^{T} x: A x=b, x \geq 0\right\} \text { and }(L D) \max \left\{b^{T} y: A^{T} y+s=c, s \geq 0\right\} \text {, }
$$

where $A \in \mathbb{R}^{m \times n}, b \in \mathbb{R}^{m}$, and $c \in \mathbb{R}^{n}$. We denote the feasible and optimal regions of the primal (dual) problem by $\mathcal{P}(\mathcal{D})$ and $\mathcal{P}^{*}\left(\mathcal{D}^{*}\right)$, respectively. Also, the strict interiors of the primal and dual feasible regions are

$$
\mathcal{P}^{o}=\{x \in \mathcal{P}: x>0\} \quad \text { and } \quad \mathcal{D}^{o}=\{(y, s) \in \mathcal{D}: s>0\} .
$$

The optimal partition, $(B \mid N)$, is defined by

$$
\begin{aligned}
B & =\left\{i: x_{i}^{*}>0, \text { for some } x^{*} \in \mathcal{P}^{*}\right\}, \text { and } \\
N & =\left\{i: s_{i}^{*}>0, \text { for some }\left(y^{*}, s^{*}\right) \in \mathcal{D}^{*}\right\} \\
& =\{1,2, \ldots, n\} \backslash B .
\end{aligned}
$$

The optimal partition is important because it characterizes the optimal sets. Allowing a set subscript on a vector (matrix) to be the subvector (submatrix) 
containing the elements (columns) in the set, we have that

$$
\begin{aligned}
\mathcal{P}^{*} & =\left\{x \in \mathcal{P}: x_{N}=0\right\}=\left\{x: A_{B} x_{B}=b, x_{B} \geq 0, x_{N}=0\right\} \text { and } \\
\mathcal{D}^{*} & =\left\{(y, s) \in \mathcal{D}: s_{B}=0\right\} \\
& =\left\{(y, s): A_{B}^{T} y=c_{B}, A_{N}^{T} y+s_{N}=c_{N}, s_{N} \geq 0, s_{B}=0\right\} .
\end{aligned}
$$

The two set partitions of $\{1,2, \ldots, n\}$ provide an algebraic description of the faces of $\mathcal{P}$. For each face $\mathcal{F}$ of $\mathcal{P}$, there is a unique partition $\left(I \mid I^{\prime}\right)$ of $\{1,2, \ldots, n\}$, such that $\mathcal{F}=\left\{x \in \mathcal{P}: x_{I} \geq 0, x_{I^{\prime}}=0\right\}$ and $\mathcal{F}^{o}=\left\{x \in \mathcal{P}: x_{I}>0, x_{I^{\prime}}=0\right\} \neq$ $\emptyset$. The dimension of $\mathcal{F}$ is $|I|-\operatorname{rank}\left(A_{I}\right)$, see [20].

The necessary and sufficient Lagrange conditions for $(L P)$ and $(L D)$ are

$$
A x=b, x \geq 0, A^{T} y+s=c, s \geq 0, \text { and } s^{T} x=0 .
$$

If the optimal partition is known, these optimality conditions may be rewritten as a linear system -i.e. without the bilinear constraint $s^{T} x=0$. This follows because if we know $(B \mid N)$, the solutions to

$$
A_{B} x_{B}=b, x_{B} \geq 0, A^{T} y=c_{B}, A^{T} y+s_{N}=c_{N} s_{N} \geq 0
$$

are in a one-to-one correspondence with the solutions to $(L P)$ and $(L D)$. For example, let $\left(x_{B}, y, s_{N}\right)$ satisfy (1). Then, setting $x_{N}=0$ and $s_{B}=0$, we have that $\left(x_{B}, x_{N}\right)$ is primal optimal and $\left(y,\left(s_{B}, s_{N}\right)\right)$ is dual optimal. Moreover, $(B \mid N)$ is the unique optimal partition of $(L P)$ and $(L D)$ if the system in (1) has a solution with $x_{B}>0$ and $s_{N}>0$.

Throughout this paper we study asymptotic linear programming, meaning that we investigate the long-term behavior of dynamic linear programs. Jeroslow $[15,16]$ was the first to study asymptotic linear programming, and several researchers have since investigated these problems $[1,2,3,23]$. This type of analysis parallels the long-term solution analysis of differential equations, but instead of investigating such things as population dynamics, asymptotic linear programs are studied to make economic interpretations $[1,3,13]$. We are interested in the dynamic, multiple-objective linear program

$$
(M O L P(t)) \min \{C(t) x: A(t) x=b(t), x \geq 0\}
$$

where $A(t): \mathbb{R} \rightarrow \mathbb{R}^{m \times n}, b(t): \mathbb{R} \rightarrow \mathbb{R}^{m}$, and $C(t): \mathbb{R} \rightarrow \mathbb{R}^{r \times n}$. The feasible region for $(M O L P(t))$ is $\mathcal{P}(t)$. Because $\mathbb{R}^{r}$ does not have a standard complete ordering for $r \geq 2$, minimization is not uniquely defined, and we use pareto optimality. A feasible solution $x$ is pareto optimal at time $t$ if there does not exist a feasible $y$ such that $C(t) y \leq C(t) x$, with strict inequality holding in at least one component. The efficient frontier at time $t$, denoted by $\mathcal{E}(t)$, is the set of all pareto optimal solutions (pareto solutions are also called efficient solutions). It is well known [9] that $x$ is efficient at time $t$ if and only if there exists a strictly positive weighting vector $w$ such that $x$ solves

$$
(L P(w, t)) \min \left\{w^{T} C(t) x: x \in \mathcal{P}(t)\right\} .
$$


The definition of the optimal partition for $M O L P(t)$, denoted by $(\stackrel{\text { molp }}{B}(t) \mid \stackrel{\text { molp }}{N}(t))$, is a natural extension of the single objective case [14], and we define

$$
\stackrel{\text { molp }}{N}(t)=\left\{i: x_{i}=0 \text { for all } x \in \mathcal{E}(t)\right\} \text { and } \stackrel{\text { molp }}{B}(t)=\{1,2, \ldots, n\} \backslash \stackrel{\text { molp }}{N}(t) .
$$

As in the single objective case, $\stackrel{\text { molp }}{N}(t)$ indexes the components of $x$ that are zero over the entire optimal set, and $\stackrel{\text { molp }}{B}(t)$ indexes the components that are positive somewhere in the optimal set. The difference is that the multiple-objective optimal partition no longer characterizes the efficient frontier. However, we do have that

$$
\mathcal{E}(t) \subseteq\left\{x \in \mathcal{P}(t): x_{N(t)}^{\operatorname{molp}_{(t)}}=0\right\}
$$

An important observation about $\stackrel{\text { molp }}{B}(t)$ is that it is the union, over the set of positive weighting vectors, of the $B$ sets of $L P(w, t)$. That is,

$$
\stackrel{\text { molp }}{B}(t)=\bigcup_{w>0}\{B:(B \mid N) \text { is the optimal partition of } L P(w, t)\} .
$$

We say that $B$ is a sub-partition of $\stackrel{\text { molp }}{B}(t)$ if there is a positive $w$ such that $(B \mid N)$ is the optimal partition of $L P(w, t)$. Other notation and terminology is consistent with the Mathematical Programming Glossary [11].

\section{Asymptotic Sign-Solvability}

The goal of this section is to develop conditions under which the collection of sign patterns of a linear system stabilize. The sign operator acts on an arbitrary matrix or vector and returns a matrix or vector of the same size whose components are replaced with the symbols,+- , and 0 . As an example,

$$
\operatorname{sign}\left(\left[\begin{array}{cccc}
-2 & 3 & 0 & 1 \\
-1 & -2 & 1 & 0
\end{array}\right]\right)=\left[\begin{array}{cccc}
- & + & 0 & + \\
- & - & + & 0
\end{array}\right] .
$$

Sign-solvability is the study of linear systems when we only know the sign patterns of the coefficient matrix and right-hand side. A large amount of research has been conducted in this area of linear algebra, and interested readers are directed to [4]. One of the more natural questions to ask is whether or not the system $M x=m$ is consistent if we only know $\operatorname{sign}(M)$ and $\operatorname{sign}(m)$. A slightly more difficult question is if there exists a solution with a fixed sign pattern. For example, if $M$ is the matrix above and $\operatorname{sign}(m)=(+,-)^{T}$, we could ask whether or not $M x=m$ has a solution with $\operatorname{sign}(x)=(0,0,+,+)^{T}($ there is no such solution). The main result of this section answers a related asymptotic question. For the functions $M: \mathbb{R} \rightarrow \mathbb{R}^{p \times q}$ and $m: \mathbb{R} \rightarrow \mathbb{R}^{p}$, does

$$
\Sigma(t)=\{\operatorname{sign}(x): M(t) x=m(t)\}
$$


stabilize? This is obviously not true for all $M$ and $m$, and we show that the conditions guaranteeing the stabilization of the optimal partition also guarantee the stabilization of $\Sigma(t)$.

The connection between sign-solvable systems and linear optimization is the necessary and sufficient Lagrange equations in (1). As previously mentioned, we know that $(B \mid N)$ is the optimal partition of $(L P)$ if and only if the linear system

$$
A_{B} x_{B}=b, \quad A_{B}^{T} y=c_{B}, \quad A_{N}^{T} y+s_{N}=c_{N}
$$

has a solution with $\operatorname{sign}\left(x_{B}\right)=(+,+, \ldots,+)^{T}$ and $\operatorname{sign}\left(s_{N}\right)=(+,+, \ldots,+)^{T}$. One way to solve a linear program is to systematically proceed through the 2 -set partitions of $\{1,2, \ldots, n\}$ and see if the above system has a positive solution. This is not an intelligent way to solve the problem as there are $2^{n}$ possible partitions, but this is a finite method to solve a linear program.

Throughout the remainder of this paper, we assume that every linear system satisfies the following assumption.

Assumption 1. For the linear system $M(t) x=m(t)$, there is a time, $T$, such that for all $t \geq T$, the determinants of all square submatrices of $[M(t) \mid m(t)]$ are either constant or have no roots. We further assume that $M(t)$ and $m(t)$ are continuous for $t \geq T$.

With this assumption, the following results were established in [13].

Lemma 2.1 (Hasfura-Beunaga, Holder, Stuart [13]). Under Assumption 1, the rank of every submatrix of $[M(t) \mid m(t)]$ stabilizes.

The next result shows that the optimal partition of a feasibility problem is constant for arbitrarily large $t$.

Lemma 2.2 (Hasfura-Beunaga, Holder, Stuart [13]). Under Assumption 1 , we have that the optimal partition of $\min \left\{0^{T} x: M(t) x=m(t), x \geq 0\right\}$ stabilizes.

As stated, Lemma 2.2 is a sub-case of the result in [13], as it only deals with the feasibility problem $\min \left\{0^{T} x: M(t) x=m(t), x \geq 0\right\}$ (the result in [13] permits a dynamic objective function as well). However, we show that dealing with the feasibility problem is enough to establish that $\Sigma(t)$ stabilizes. For a sign pattern $\sigma$, we let

$$
\Lambda_{+}(\sigma)=\left\{i: \sigma_{i}=+\right\}, \Lambda_{-}(\sigma)=\left\{i: \sigma_{i}=-\right\} \text {, and } \Lambda_{0}(\sigma)=\left\{i: \sigma_{i}=0\right\} .
$$

So, if $\sigma=(+,-,+,+, 0)$, we have that $\Lambda_{+}(\sigma)=\{1,3,4\}, \Lambda_{-}(\sigma)=\{2\}$, and $\Lambda_{0}(\sigma)=\{5\}$. Theorem 2.3 shows that there is a time after which $\Sigma(t)$ is constant.

Theorem 2.3. Under Assumption 1, we have that there is a time $T$ such that for all $t \geq T, \Sigma(t)=\Sigma(T)$. 
Proof. Let $t_{0}$ be large enough to guarantee that for $t \geq t_{0}$ the ranks of the submatrices of $[M(t) \mid m(t)]$ are constant and that the optimal partition of $\min \left\{0^{T} x\right.$ : $M(t) x=m(t), x \geq 0\}$ is constant. If $\operatorname{rank}\left(M\left(t_{0}\right)\right) \neq \operatorname{rank}\left(\left[M\left(t_{0}\right) \mid m\left(t_{0}\right)\right]\right)$, we have that $\Sigma(t)=\emptyset$ for all $t \geq t_{0}$.

Assume that $\operatorname{rank}(M(t))=\operatorname{rank}([M(t) \mid m(t)])$, for all $t \geq t_{0}$, and let $\sigma \in$ $\Sigma\left(t_{0}\right)$. Consider the optimization problem,

$$
\begin{aligned}
& \min \left\{0^{T} x: M\left(t_{0}\right) x=m\left(t_{0}\right), \operatorname{sign}(x)=\sigma\right\} \\
& \quad=\min \left\{0^{T} x: M\left(t_{0}\right) x=m\left(t_{0}\right), x_{\Lambda_{+}(\sigma)}>0, x_{\Lambda_{-}(\sigma)}<0, x_{\Lambda_{0}(\sigma)}=0\right\} .
\end{aligned}
$$

Let $P$ be the $n \times n$ diagonal matrix such that $P_{(i, i)}=-1$ if $i \in \Lambda_{-}(\sigma)$, and $P_{(i, i)}=1$ otherwise. Then, if $z=P x$, we have that

$$
\begin{aligned}
& \operatorname{argmin}\left\{0^{T} x: M\left(t_{0}\right) x=m\left(t_{0}\right), x_{\Lambda_{+}(\sigma)}>0, x_{\Lambda_{-}(\sigma)}<0, x_{\Lambda_{0}(\sigma)}=0\right\} \\
& \quad=\operatorname{argmin}\left\{0^{T} z: M\left(t_{0}\right) P z=m\left(t_{0}\right), z_{\Lambda_{+}(\sigma)}>0, z_{\Lambda_{-}(\sigma)}>0, z_{\Lambda_{0}(\sigma)}=0\right\} .
\end{aligned}
$$

The optimization problem in (2) has a solution if and only if the optimal partition to the linear program

$$
\min \left\{0^{T} x: M\left(t_{0}\right) P z=m\left(t_{0}\right), z_{\Lambda_{0}(\sigma)}=0, z \geq 0\right\}
$$

is $\left(\Lambda_{+}(\sigma) \cup \Lambda_{-}(\sigma) \mid \Lambda_{0}(\sigma)\right)=\left(B\left(t_{0}\right) \mid N\left(t_{0}\right)\right)$. From the fact that $\sigma$ is in $\Sigma\left(t_{0}\right)$, we know that the optimization problem in (2) has a solution, and hence, the optimal partition of the linear problem in (3) is $\left(\Lambda_{+}(\sigma) \cup \Lambda_{-}(\sigma) \mid \Lambda_{0}(\sigma)\right)$. From Lemma 2.2 we have that this optimal partition is constant for every $t \geq t_{0}$. So, the optimization problem in (2) has a solution for every $t \geq t_{0}$, which means that $\sigma \in \Sigma(t)$ for every $t \geq t_{0}$.

So far we have established that $\Sigma\left(t_{0}\right) \subseteq \Sigma(t)$, for $t \geq t_{0}$. If at some time $t_{1}>t_{0}$ a new sign pattern enters $\Sigma\left(t_{1}\right)$, the same reasoning shows that this new sign pattern is in $\Sigma(t)$, for all $t \geq t_{1}$. Since there are $3^{n}$ sign patterns, we know that there is a time $T$ such that $\Sigma(t)=\Sigma(T)$, for all $t \geq T$.

\section{Stabilization of the Multiple Objective Opti- mal Partition}

In this section we show that the multiple objective optimal partition stabilizes. Let $\left\{\left(B^{1} \mid N^{1}\right),\left(B^{2} \mid N^{2}\right), \ldots,\left(B^{2^{n}} \mid N^{2^{n}}\right)\right\}$ be the collection of two-set partitions of $\{1,2, \ldots, n\}$. For $i=1,2, \ldots, 2^{n}$, we let

$$
H_{i}(t)=\left[\begin{array}{cccc}
A_{B^{i}}(t) & 0 & 0 & 0 \\
0 & A_{B^{i}}^{T} & -C_{B^{i}}^{T}(t) & 0 \\
0 & A_{N^{i}}^{T} & -C_{N^{i}}^{T}(t) & I_{\left|N^{i}\right|}
\end{array}\right], h(t)=\left(\begin{array}{c}
b(t) \\
0 \\
0
\end{array}\right) \text {, and } v=\left(\begin{array}{c}
x_{B^{i}} \\
y \\
w \\
s_{N^{i}}
\end{array}\right) \text {. }
$$

We say that $v$ is sufficiently non-negative (sufficiently positive), written $v \searrow_{0}$ $(v>0)$, if $x_{B^{i}}, w$, and $s_{N^{i}}$ are non-negative (positive). So, for any partition 
$\left(B^{i} \mid N^{i}\right)$ we have that the Lagrange conditions of $L P(w, t)$ are the same as the consistency of $H_{i}(t) v=h(t), v \unlhd 0$. We also have that $B^{i}$ is a sub-partition of $\stackrel{\text { molp }}{B}(t)$ if and only if $H_{i}(t) v=h(t), v>10$ is consistent.

Remember that for each $i$ we assume the system $H_{i}(t) v=h(t)$ satisfies Assumption 1. We point out that the class of functions described by Assumption 1 includes the rational and transcendental functions. In fact, sin and cos are permitted so long as they are shifted to satisfy the condition of not having zeros. For example, Assumption 1 is satisfied by

$$
A(t)=\left[\begin{array}{cc}
1 / t & t^{2} \\
e^{t} & \sin (t)+\ln (t)
\end{array}\right], \quad C(t)=\left[\begin{array}{cc}
t & t+\sin (t) \\
1 / \sqrt{t} & 1
\end{array}\right], \quad \text { and } \quad b(t)=\left(\begin{array}{c}
e^{-t} \\
t^{3}
\end{array}\right) .
$$

As in [13], one of the strengths of our proof technique is that it permits a broader class of functions than what was previously available, with rational functions being required in [15] and [16] and linear functions being required in [1], [2], [3], and [23]. The following result states that the multiple objective optimal partition stabilizes over this larger class of functions.

Theorem 3.1. There exists a time $T$, such that for all $t \geq T,\left(\left.\stackrel{\text { molp }}{B}(t)\right|^{\text {molp }} N(t)\right)=$ $(\stackrel{\text { molp }}{B}(T) \mid \stackrel{\text { molp }}{N}(T))$.

Proof. For $i=1,2, \ldots, 2^{n}$, let

$$
\stackrel{\text { suff }}{\Sigma_{i}}(t)=\left\{\operatorname{sign}(v): H_{i}(t) v=h(t), v>0\right\} \quad \text { and } \quad \Omega(t)=\left\{i: \Sigma_{i}^{\text {suff }}(t) \neq \emptyset\right\} .
$$

So, at any time we see that

$$
\stackrel{\text { molp }}{B}(t)=\bigcup_{i \in \Omega(t)} B^{i}
$$

From Theorem 2.3 we have that each $\Sigma_{i}^{\text {suff }}(t)$ becomes constant, and hence, there is a time $T$ after which every $\Sigma_{i}(t)$ is constant. So, the index set in (4) stabilizes, and consequently, so does $\stackrel{\text { molp }}{B}(t)$.

The asymptotic, multiple objective optimal partition is denoted by $(\stackrel{\text { molp }}{B \mid N o l p} N)$, with the dependence on time being removed. From Theorem 3.1 we see that there is point in time at which the collection of variables that are zero in every efficient solution is constant. We point out that it may not be possible for all of the remaining variables to be positive in an efficient solution. In fact, this can only happen if the efficient frontier stabilizes to a single face (an unlikely event). The geometric interpretation of Theorem 3.1 is that as time increases without bound, the 'shape', dimension, and number of faces of the efficient frontier stabilize. 
We now establish that the efficient frontier is continuous for arbitrarily large time. The efficient frontier is a point-to-set map that depends on $t$, and such maps are continuous at $t^{0}$ (in the Hausdorff sense [12]) if, for any sequence $t^{k} \rightarrow t^{0}$, we have that

1. if $x^{0}$ is in $\mathcal{E}\left(t^{0}\right)$, then there is a sequence $x^{k}$ in $\mathcal{E}\left(t^{k}\right)$ that converges to $x^{0}$, and

2. if $x^{k}$ is a sequence in $\mathcal{E}\left(t^{k}\right)$ that converges to $x^{0}$, then $x^{0}$ is in $\mathcal{E}\left(t^{0}\right)$.

Our proof relies on the following previously established results.

Lemma 3.2 (Campbell and Meyer [5]). The Moore-Penrose pseudo inverse of $M: \mathbb{R} \rightarrow \mathbb{R}^{p \times q}$ is continuous at $t_{0}$ if and only if $\operatorname{rank}(M(t))=\operatorname{rank}\left(M\left(t_{0}\right)\right)$, for $t$ sufficiently close to $t_{0}$.

The next result shows that the analytic center of a polytope is continuous if the Moore-Penrose pseudo inverse of the coefficient matrix is continuous. At any time $t$, the analytic center of $\mathcal{M}(t)=\{x: M(t) x=m(t), x \geq 0\}$ is the unique solution to

$$
\max \left\{\sum_{i=1}^{n} \ln (x): x \in \mathcal{M}^{o}(t)\right\},
$$

which is only defined if $\mathcal{M}$ is bounded and $\mathcal{M}^{\circ}$ is non-empty.

Lemma 3.3 (Caron and Holder [6]). Assume that $\mathcal{M}(t)$ is bounded and that the strict interior of $\mathcal{M}(t)$ is non-empty. Also assume that $M(t)$ and $m(t)$ are continuous. Then, the analytic center of $\mathcal{M}(t)$ is continuous at $t^{0}$ if the Moore-Penrose pseudo inverse of $M(t)$ is continuous at $t_{0}$.

To use Lemma 3.3 we need the assumption that $\mathcal{P}^{o}$ is non-empty. This assumption is equivalent to assuming that the dual optimal set is bounded [20]. Such an assumption is natural in light of Hausdorff convergence because Hausdorff topologies naturally yield the notion of compactness, a property that we are assuming about the dual faces. The proof of Theorem 3.4 requires a convergent dual sequence, which is possible because the dual optimal sets are assumed to be compact.

Theorem 3.4. The efficient frontier is continuous for arbitrarily large $t$, provided that $\mathcal{P}^{o}(t) \neq \emptyset$.

Proof. Let $t^{0}$ be large enough so that for some $\delta>0$, we have for all $t \geq t^{0}-\delta$ that $(\stackrel{\text { molp }}{B} \mid \stackrel{\text { molp }}{N})=(\stackrel{\text { molp }}{B}(t) \mid \stackrel{\text { molp }}{N}(t))$ and that the ranks of all submatrices of $A(t)$ are constant. Let $t^{k} \rightarrow t^{0}$, and assume that $x^{0}$ is in $\mathcal{E}\left(t^{0}\right)$. Then, $x^{0}$ is in the strict interior of a unique face of the efficient frontier, and hence, there is a unique partition $\left(B^{i} \mid N^{i}\right)$ such that for some $y^{0}, w^{0}$, and $s_{N^{i}}^{0}$, we have that $v\left(t^{0}\right)=\left(x_{B^{i}}^{0}, y^{0}, w^{0}, s_{N^{i}}^{0}\right)^{T}$ is a solution to $H_{i}\left(t^{0}\right) v=h_{i}\left(t^{0}\right), v>0$. Thus, $v\left(t^{0}\right)=$ $H_{i}^{+}\left(t^{0}\right) h\left(t^{0}\right)+q^{0}$, where $H_{i}^{+}\left(t^{0}\right)$ is the Moore-Penrose pseudo inverse of $H_{i}\left(t^{0}\right)$ 
and $q^{0} \in \operatorname{Null}\left(H_{i}\left(t^{0}\right)\right)$. We define $v\left(t^{k}\right)=H_{i}^{+}\left(t^{k}\right) h\left(t^{k}\right)+\left(I-H_{i}^{+}\left(t^{k}\right) H_{i}\left(t^{k}\right)\right) q^{0}$. Then,

$$
\begin{aligned}
H_{i}\left(t^{k}\right) v\left(t^{k}\right) & =H_{i}\left(t^{k}\right)\left[H_{i}^{+}\left(t^{k}\right) h\left(t^{k}\right)+\left(I-H_{i}^{+}\left(t^{k}\right) H_{i}\left(t^{k}\right)\right) q^{0}\right] \\
& =H_{i}\left(t^{k}\right) H_{i}^{+}\left(t^{k}\right) h\left(t^{k}\right)+H_{i}\left(t^{k}\right) q^{0}-H_{i}\left(t^{k}\right) H_{i}^{+}\left(t^{k}\right) H_{i}\left(t^{k}\right) q^{0} \\
& =h\left(t^{k}\right)-H_{i}\left(t^{k}\right) q^{0}+H_{i}\left(t^{k}\right) q^{0} \\
& =h\left(t^{k}\right),
\end{aligned}
$$

where the $3^{\text {rd }}$ equality holds because $h\left(t^{k}\right)$ is in the column space of $H_{i}\left(t^{k}\right)$.

Since $\left(I-H_{i}^{+}\left(t^{0}\right) H_{i}\left(t^{0}\right)\right) q^{0}$ is the projection of $q^{0}$ onto the null space of $H_{i}\left(t^{0}\right)$ and $q^{0} \in \operatorname{Null}\left(H_{i}\left(t^{0}\right)\right)$, we see that $\left(I-H_{i}^{+}\left(t^{0}\right) H_{i}\left(t^{0}\right)\right) q^{0}=q^{0}$. From Lemmas 2.1 and 3.2 we have that $H_{i}^{+}(t)$ is continuous. So, as $t^{k} \rightarrow t^{0}$,

$$
v\left(t^{k}\right)=H_{i}^{+}\left(t^{k}\right) h\left(t^{k}\right)+\left(I-H_{i}^{+}\left(t^{k}\right) H_{i}\left(t^{k}\right)\right) q^{0} \rightarrow H_{i}^{+}\left(t^{0}\right) h\left(t^{0}\right)+q_{0}=v\left(t^{0}\right)>0 .
$$

Notice that $v\left(t^{k}\right)>0$ for large $k$. Setting $x_{N^{i}}^{k}=0$, we have that $x^{k}$ is in $\mathcal{E}\left(t^{k}\right)$ and that it converges to $x^{0}$. Hence, we have established the first condition of continuity.

Assume that $x^{k}$ in $\mathcal{E}\left(t^{k}\right)$ converges to $x^{0}$. Since each $x^{k}$ is in the strict interior of a unique face of $\mathcal{E}\left(t^{k}\right)$, we have that each $k$ corresponds to a unique optimal partition. Since there are a finite number of partitions, we assume without loss of generality that $\left(B^{1} \mid N^{1}\right),\left(B^{2} \mid N^{2}\right), \ldots,\left(B^{s} \mid N^{s}\right)$ are the optimal partitions observed an infinite number of times. For $j=1,2, \ldots, s$, we let $k_{j}$ be the subsequence of $k$ such that $\left(B^{j} \mid N^{j}\right)$ is the optimal partition at time $t^{k_{j}}$. For any $k_{j}$, there exists a positive $w^{k_{j}}$ and a dual feasible $\left(y^{k_{j}}, s^{k_{j}}\right)$ such that $v\left(t^{k_{j}}\right)=$ $\left(x_{B^{j}}^{k_{j}}, y^{k_{j}}, w^{k_{j}}, s_{N^{j}}^{k_{j}}\right)^{T}$ is a sufficiently positive solution to $H_{j}\left(t^{k_{j}}\right) v=h\left(t^{k_{j}}\right)$. The proof would be complete if $v\left(t^{k_{j}}\right)$ converged, but we only have that the subvector $x_{B^{j}}^{k}$ converges. We select the remaining components to guarantee convergence. First, we assume without loss of generality that $\left\|w^{k}\right\|=1$, and hence, $w^{k}$ has a convergent subsequence. For simplicity, we assume that $w^{k_{j}} \rightarrow w^{j}$. Second, we select $\left(y^{k_{j}}, s^{k_{j}}\right)$ to be the analytic center of

$$
\left\{(y, s): A_{B^{j}}^{T}\left(t^{k_{j}}\right) y=C_{B^{j}}^{T}\left(t^{k_{j}}\right) w^{k_{j}}, A_{N^{j}}^{T}\left(t^{k_{j}}\right) y+s_{N^{j}}=C_{N^{j}}^{T}\left(t^{k_{j}}\right) w^{k_{j}}, s_{N^{j}} \geq 0\right\} .
$$

So, $\left(y^{k_{j}}, s^{k_{j}}\right)$ is the analytic center of the optimal face of the dual of $L P\left(w^{k_{j}}, t^{k_{j}}\right)$. Notice that $t^{0}$ is selected to be large enough to guarantee that for each $j$ and $t \geq t^{0}-\delta$ that

$$
\operatorname{rank}\left(\left[\begin{array}{ll}
A_{B^{j}}^{T}(t) & 0 \\
A_{N^{j}}^{T}(t) & I
\end{array}\right]\right)=\operatorname{rank}\left(\left[\begin{array}{ll}
A_{B^{j}}^{T}\left(t^{0}\right) & 0 \\
A_{N^{j}}^{T}\left(t^{0}\right) & I
\end{array}\right]\right)
$$

From Lemmas 3.3 and 3.2 we have that $\left(y^{k_{j}}, s^{k_{j}}\right)$ converges to the analytic center of

$$
\left\{(y, s): A_{B^{j}}^{T}\left(t^{0}\right) y=C_{B^{j}}^{T}\left(t^{0}\right) w^{0}, A_{N^{j}}^{T}\left(t^{0}\right) y+s_{N^{j}}=C_{N^{j}}^{T}\left(t^{0}\right) w^{0}, s_{N^{j}} \geq 0\right\} .
$$

We now have for any $j$ that $v\left(t^{k_{j}}\right)=\left(x_{B^{j}}^{k_{j}}, w^{k_{j}}, y^{k_{j}}, s_{N^{j}}^{k_{j}}\right)$ converges, say to $v^{0}$. Since $H_{j}\left(t^{0}\right) v^{0}=h\left(t^{0}\right)$ and $v^{0} \searrow_{0}$, we see that $x^{0} \in \mathcal{E}\left(t^{0}\right)$. 
Theorem 3.4 adds to our geometric intuition by showing that the efficient frontier is altered continuously as time grows without bound. From Theorems 3.1 and 3.4 we now have for large $t$ that 1 ) the number of faces in the efficient frontier is constant, 2) the dimension of each face is constant, and 3) the faces are altered in a continuous fashion. In fact, the proof of Theorem 3.4 shows that a continuous solution exists for every sub-partition of $\stackrel{\text { molp }}{B}$.

Corollary 3.5. Let $B^{i}$ be a sub-partition of $B$. Then, for large $t$ there is a continuous $(x(t), w(t))$ such that

$x_{B^{i}}(t)>0, \quad x_{N^{i}}(t)=0, \quad$ and $\quad x(t) \in \operatorname{argmin}\left\{w^{T}(t) C(t) x: A(t) x=b(t), x \geq 0\right\}$.

Proof. The proof follows directly from the first part of the proof of Theorem 3.4. Notice that this part of the proof does not require that $\mathcal{P}^{o} \neq \emptyset$.

We conclude this section by investigating the time at which the optimal partition stabilizes. For any weighting vector $w$, let $\left(B_{w}(t) \mid N_{w}(t)\right)$ be the optimal partition of $L P(w, t)$ and define

$$
T(w)=\inf \left\{T: \text { for all } t>T,\left(B_{w}(t) \mid N_{w}(t)\right)=\left(B_{w}(T) \mid N_{w}(T)\right)\right\} .
$$

This function maps the weighting vector, $w$, to the time when the optimal partition of $L P(w, t)$ stabilizes. In [14], it is shown that for any fixed $w$, the optimal partition of $L P(w, t)$ stabilizes, so $T(w)$ exists for all $w>0$. We originally attempted to prove Theorem 3.1 by finding an upper bound on $T(w)$, but this technique is flawed, as shown in the following example.

Example 3.6. Let

$$
c(t)=\left[\begin{array}{rr}
-1 & 0 \\
0 & -t
\end{array}\right], A(t)=\left[\begin{array}{llll}
2 & 3 & 1 & 0 \\
3 & 2 & 0 & 1
\end{array}\right], \text { and } b(t)=\left(\begin{array}{l}
6 \\
6
\end{array}\right) .
$$

Without loss of generality, we assume that $\|w\|=1$. Then, $L P(w, t)$ is

$$
\begin{aligned}
\max \{ & \left(1-w_{2}\right) x_{1}+w_{2} t x_{2}: \\
& \left.2 x_{1}+3 x_{2}+x_{3}=6,3 x_{1}+2 x_{2}+x_{4}=6, x_{i} \geq 0, i=1,2,3,4\right\},
\end{aligned}
$$

where $w_{2}$ varies in $(0,1)$. It is easy to see that for all $t>0,\left(\left.\stackrel{\text { molp }}{B}(t)\right|^{\text {molp }} N(t)\right)=$ $(\{1,2,3,4\} \mid \emptyset)$. However, the partition of $L P(w, t)$ stabilizes when $\left(1-w_{2}\right)=w_{2} t$, and hence, we have that $T(w)=\left(1-w_{2}\right) / w_{2}$. Clearly this function is not bounded over the interval $(0,1)$.

Example 3.6 helps us interpret Theorem 3.1. The fact that the multiple objective optimal partition stabilizes does not mean that there is a time after which the optimal partitions of the single objective programs $L P(w, t)$ are constant. However, it does mean that there is a time after which the optimal partition of $L P(w, t)$ provides a sub-partition of $\stackrel{\text { molp }}{B}$. Hence, there is a time, independent of 
$w$, after which we know that the solutions to $L P(w, t)$ are efficient, even if the optimal partition of this single objective program has not stabilized.

While $T(w)$ is not in general bounded, it is quasi-convex over the weighting regions that define an optimal partition. For each $\left(B^{i} \mid N^{i}\right)$, we let

$$
V^{i}=\left\{w>0:\left(B^{i} \mid N^{i}\right) \text { is the asymptotic optimal partition of } L P(w, t)\right\} .
$$

The following results shows that each $V^{i}$ is convex and that $T(w)$ is quasiconvex.

Theorem 3.7. For each $i=1,2, \ldots, 2^{n}$, we have that $V^{i}$ is convex. Moreover, $T$ is a quasi-convex function over $V^{i}$.

Proof. Let $\left\{w^{1}, w^{2}, \cdots, w^{p}\right\} \subseteq V^{i}$ and let $t \geq \max \left\{T\left(w^{j}\right): j=1, \ldots, p\right\}$. Then, for $j=1, \ldots, p$, there exist $x, y^{j}$, and $s^{j}$ such that

$$
\begin{aligned}
A_{B^{i}}(t) x_{B^{i}} & =b(t), x_{B^{i}}>0 \\
A_{N^{i}}^{T}(t) y^{j}+s_{N^{i}}^{j} & =C_{N^{i}}^{T}(t) w^{j}, s_{N^{i}}^{j}>0, \text { and } \\
A_{B^{i}}^{T}(t) y^{j} & =C_{B^{i}}^{T}(t) w^{j} .
\end{aligned}
$$

Let $w=\sum_{j} \alpha_{j} w^{j}$, where $\sum_{j} \alpha_{j}=1$ and $\alpha_{j} \geq 0$. Allowing $y=\sum_{j} \alpha_{j} y^{j}$ and $s=\sum_{j} \alpha_{j} s^{j}$, we see that

$$
\begin{aligned}
A_{B^{i}}(t) x_{B^{i}} & =b(t), x_{B^{i}}>0 \\
A_{N^{i}}^{T}(t) y+s_{N^{i}} & =C_{N^{i}}^{T}(t) w, s_{N^{i}}>0, \text { and } \\
A_{B^{i}}^{T}(t) y & =C_{B^{i}}^{T}(t) w .
\end{aligned}
$$

Since these are the necessary and sufficient conditions for $\left(B^{i} \mid N^{i}\right)$ to be the optimal partition of $L P(w, t)$, we have that $V^{i}$ is convex. Moreover, the choice of $t$ was arbitrary past $\max \left\{T\left(w^{j}\right): j=1, \ldots, p\right\}$. So, $T(w) \leq \max \left\{T\left(w^{j}\right)\right.$ : $j=1, \ldots, p\}$, and $T$ is quasi-convex.

\section{Economic Interpretations}

In this section, we demonstrate the usefulness of Theorem 3.1 by showing how it extends the economics insights of the Nonsubstitution Theorem. Suppose that at any given time the economy transforms input commodities and labor into output commodities. Suppose further that the entire economy has $n$ commodities produced by $m$ processes and $r$ labor sources. Under some typical economic assumptions, we determine the price of each commodity and a production schedule that indicates how processes are to be used.

We assume that the available technology allows constant returns to scale, meaning that changing the inputs to a process by some factor changes the outputs by the same factor. This hypothesis is the subject of constant debate in economics, but it is not unreasonable. After all, if we have one factory producing a commodity, should we not expect to produce twice the amount if we purchase 
an identical factory? The assumption of constant returns to scale means that for every time $t$, every process $i$, and every commodity $j$, there is a real number $a_{j}^{i}(t)$ equal to the number of units of commodity $j$ required to run process $i$ at unit intensity. Similarly, let $b_{j}^{i}(t)$ be the number of units of commodity $j$ yielded by a unit of process $i$, and $l_{k}^{i}(t)$ be the number of units of labor type $k$ required to use process $i$ at unit intensity. We assume that labor is the only nonproducible commodity and that no process operates free of labor. So, for each process $i$ there exists some labor type $k$ such that $l_{k}^{i}(t)$ is positive. For every time $t$, we construct the matrices $A(t), B(t)$, and $L(t)$ with entries $a_{j}^{i}(t)$, $b_{j}^{i}(t), l_{k}^{i}(t)$, with the rows being indexed by $i$ and the columns by $j$ or $k$. We represent a choice of prices, wages, and process utilization by the vectors $p, w$, $x$, respectively. With this notation, we see that at time $t$ the total amount spent on running the processes is $x^{T}(A(t)+L(t)) p$ and that the aggregate earnings from the output commodities is $x^{T} B(t) p$.

In elementary economic models, the prevailing rate of interest on loaned money is derived from the particular climate of risk in the economy, and in turn, this interest rate induces a maximum rate of profit. We assume that if some industry yields a higher rate of profit than the interest rate, then new firms will enter the industry and increases the supply, which in turn lowers the market price and the rate of profit. Thus, we assume that there is a maximum rate of profit that any choice of prices, wages, and use of capital may yield. For each time $t$, we let $r(t)$ be the rate of maximum profit associated with the economy at time $t$. A process that does not achieve the maximum rate of profit incurs extra costs. Similarly, a process that yields a higher rate of profit than $r(t)$ provides extra profits. Our assumption of a maximum marginal profit does not allow a process to yield extra profits. This means that for any prices and wages we have that

$$
[B(t)-(1+r(t)) A(t)] p \leq L(t) w
$$

Moreover, it is natural to assume that only process achieving the maximum rate will be selected, as underachieving process will not attract investment. The algebraic representation of this assumption is

$$
x^{T}[B(t)-(1+r(t)) A(t)] p=x^{T} L(t) w .
$$

We require that each commodity be the sole product of at least one process, but some processes are allowed to produce multiple commodities. Processes that produce multiple commodities are not permitted to yield a savings in input commodities over the most efficient processes that produces the commodities individually. However, multiple output processes are allowed to make more efficient use of labor. This assumption is a weakening of the standard economic assumption called single production, which requires that all processes produce a single commodity. We do not go as far as allowing joint production, which allows more efficient use of input commodities and labor. We call our assumption joint production without commodity savings. This assumption imposes a structure on $A$ and $B$. We scale input and output units so that quantities are in terms of a 
single output unit. This means that $B$ is a 0,1 matrix, with $b_{j}^{i}=1$ meaning that process $i$ makes commodity $j$. If we assumed single production, then each row of $B$ would contain a single 1 . However, the assumption of joint production without commodity savings allows the rows of $B$ to contain several 1s, but only if $A$ has a special structure. Let the $i^{\text {th }}$ row of $B$ have a 1 in column $j^{\prime}$ and $j^{\prime \prime}$ -i.e process $i$ makes commodities $j^{\prime}$ and $j^{\prime \prime}$. Let $i_{1}^{\prime}, i_{2}^{\prime}, \ldots, i_{u}^{\prime}$ and $i_{1}^{\prime \prime}, i_{2}^{\prime \prime}, \ldots, i_{v}^{\prime \prime}$ be the process that uniquely produce commodity $j^{\prime}$ and $j^{\prime \prime}$. The assumption of joint production without commodity savings requires that for some $i_{p}^{\prime}$ and $i_{q}^{\prime \prime}$, the $i^{\text {th }}$ row of $A$ is the sum of the $i_{p}^{\prime}$ and $i_{q}^{\prime \prime}$ rows of $A$. So, with respect to commodity inputs process $i$ replicates processes $i_{p}^{\prime}$ and $i_{q}^{\prime \prime}$. The difference between using process $i$ and jointly using processes $i_{p}^{\prime}$ and $i_{q}^{\prime \prime}$ is that the labor requirements for process $i$ may differ from the labor requirements of jointly using processes $i_{p}^{\prime}$ and $i_{q}^{\prime \prime}$.

We have not yet mentioned anything about monetary units, and without one prices are only relative. It is convenient to specify a standard of value, called a numeraire in the language of economics. Specifically, we let the numeraire be the collection of commodities that satisfy demand, and we assume that prices are scaled so that the total value of the numeraire is one monetary unit. So, allowing $d(t)$ to be the positive vector of demands at time $t$, we require that prices are scaled to satisfy $d(t)^{T} p=1$.

The economic model that we consider is

$$
\begin{aligned}
{[B(t)-(1+r(t)) A(t)] p } & \leq L(t) w \\
x^{T}[B(t)-(1+r(t)) A(t)] p & =x^{T} L(t) w \\
x^{T} B(t) & >0 \\
d(t)^{T} p & =1 \\
x, p & \geq 0 \\
w & >0 .
\end{aligned}
$$

The first two constraints make sure that no process earns extra profits and that the processes that are used attain the maximum profit. The third constraint guarantees that every commodity is produced, and the fourth equality defines a monetary unit. A choice of prices, $p$, a wage structure, $w$, and a manner to operate the processes, $x$, satisfying this system is an equilibrium point of the economy. The goal of equilibrium models is to show that in an economy, prices, wages, and industrial production are not random but are subject to governing forces, guided, in Adam Smith's phrase, by an invisible hand [22]. Our goal is to show that the operating structure of an economy in equilibrium stabilizes independent of how labor is paid.

Kurz and Salvadori [18] show that if we assume single production and a single labor source (so $L$ has a single column and $w$ is a scalar instead of a vector), then this economic model has an equilibrium solution if and only if the following dual pair of linear programs is consistent,

$$
\begin{array}{r}
\min \left\{L(t)^{T} x: x^{T}[B(t)-(1+r(t)) A(t)] \geq d(t)^{T}, x \geq 0\right\}, \text { and } \\
\max \left\{d(t)^{T} y:[B(t)-(1+r(t)) A(t)] y \leq L(t), y \geq 0\right\} .
\end{array}
$$


The first linear program has the simple interpretation of minimizing labor while meeting demand. If $x^{*}(t)$ and $y^{*}(t)$ are optimal for (6) and (7), then $x(t)=x^{*}(t), p(t)=y^{*}(t) /\left(d(t)^{T} y^{*}(t)\right)$, and $w(t)=1 /\left(d(t)^{T} y^{*}(t)\right)$ are equilibrium solutions of (5). The Nonsubstitution Theorem, stated in mathematical programming terms, is below.

Theorem 4.1 (Nonsubstitution Theorem [21]). Assume that $B(t), A(t)$, $L(t)$, and $r(t)$ are constant functions and that $L(t)$ has a single column. Then, there is a basic optimal solution of (6) that is optimal independent of $d(t)$.

In economics terms, this result states that there is a collection of processes that yields an economy in equilibrium and that this collection does not depend on the demands of the economy. We point out that the Nonsubstitution Theorem does not say the collection of processes is unique, and it is easy to construct examples where there are several such collections (each corresponding to a different optimal basis).

Hasfura-Buenaga, Holder, and Stuart [13] extend this result in several ways. First, they show that solving the linear programs in (6) and (7) is the same as finding an equilibrium solution of (5) under the assumption of joint production without commodity savings. Second, they establish that as long as there is a single labor source, the optimal partition of (6) stabilizes (assuming that the Lagrange conditions defined by the functions $A(t), B(t), L(t), r(t)$, and $d(t)$ satisfy Assumption 1). This economic extension means that the entire collection of processes that can be used in an economy in equilibrium becomes constant. The result is true even when the technology of the processes, the demand for the commodities, and the interest rate are allowed to dynamically vary with time.

We keep the assumption of joint production without commodity savings but examine the more realistic situation where labor is heterogeneous -i.e. where there are $r$ labor sources. The immediate extension to multiple labor sources is that the economy in (5) has an equilibrium solution with wage structure $w$ if and only if the following linear programs are consistent.

$$
\begin{array}{r}
\min \left\{w^{T} L(t)^{T} x: x^{T}[B(t)-(1+r(t)) A(t)] \geq d(t)^{T}, x \geq 0\right\}, \text { and } \\
\max \left\{d(t)^{T} y:[B(t)-(1+r(t)) A(t)] y \leq L(t) w, y \geq 0\right\} .
\end{array}
$$

Considering all positive wage vectors $w$, the solutions to (8) form the efficient frontier of the multiple objective linear program

$$
\min \left\{L(t)^{T} x: x^{T}[B(t)-(1+r(t)) A(t)] \geq d(t)^{T}, x \geq 0\right\} .
$$

An immediate corollary to Theorem 3.1 is that the multiple objective optimal partition stabilizes, and hence, for any wage structure we know that the entire collection of processes that can be used to achieve an equilibrium stabilizes. This is formalized in the following theorem.

Theorem 4.2. Let $A(t), B(t), L(t), r(t)$, and $d(t)$ be such that Theorem 3.1 applies to the the multiple objective program in (10). Then, under the assumption of joint production without commodity savings, there is a time $T$, independent 
of the wage structure, for which the collection of processes that are capable of achieving economic equilibrium is constant for all $t \geq T$.

The important aspect of Theorem 4.2 is that $T$ is independent of the wage structure $w$. So, in an economy where technology, demand, and the interest rate are dynamic, we have that there is a time after which the collection of optimal processes is constant no matter how labor is paid. Hence, we have the surprising economic result that the wage structure does not effect the long term behavior of the model.

\section{Conclusion}

There are three primary contributions in this work. The first of these is stated in Theorem 2.3, where we show that the sign patterns of a dynamic linear system stabilize. This result has a different flavor than the previous combinatorial work on sign-solvable systems. The second main result shows that the MOLP optimal partition stabilizes. This is not surprising because the analogous single objective result was already known. However, the proof of our extension hinges on the sign pattern stabilization of a dynamic linear system, and a straight forward extension of the single objective case showing that $T(w)$ is bounded is not possible. Finally, we used this result to extend a dynamic version of the Nonsubstitution Theorem in a way that allows multiple labor sources.

\section{References}

[1] E. Altman, K. Avrachenkov, and J. Filar. Asymptotic linear programming and policy improvement for singularly perturbed markov decision processes. Mathematical Methods of Operations Research, 59(1):97-109, 1999.

[2] L. Bernard. A generalized inverse method for asymptotic linear programming. Mathematical Programming, 43(1):71-86, 1989.

[3] L. Bernard. An efficient basis update for asymptotic linear programming. Linear Algebra and its Applications, 184:83-102, 1993.

[4] R. Brualdi and B. Shader. Matrices and Sign-Solvable Linear Systems. Cambridge University Press, New York, NY, 1995.

[5] S. Campbell and Jr. C. Meyer. Generalized Inverses of Linear Transformations. Fearon Pitman Publishers Inc., Belmont, CA, 1979.

[6] R. Caron, H. Greenberg, and A. Holder. Analytic centers and repelling inequalities. Technical Report CCM 142, Center for Computational Mathematics, University of Colorado at Denver, 1999. To appear in European Journal of Operations Research.

[7] P. Chander. A simple proof of the nonsubstitution theorem. Quaterly Journal of Economics, 88:698-701, 1974. 
[8] W. Diewart. The samuelson nonsubstitution theorem and the computation of equilibrium prices. Econometrica, 43(1):57-64, 1975.

[9] M. Ehrgott. Multicriteria Optimization, volume 491 of Lecture Notes in Economics and Mathematical Systems. Springer, New York, NY, 2000.

[10] T. Fujimoto. A simple proof of the nonsubstitution theorem. Journal of Quantitative Economics, 3(1):35-38, 1987.

[11] H. Greenberg. Mathematical Programming Glossary. World Wide Web, http://www-math. cudenver . edu/ hgreenbe/glossary/glossary. html, 1996-2001.

[12] B. Grunbaum. Convex Polytopes. Wiley-Interscience, New York, NY, 1967.

[13] J. Hasfura-Buenaga, A. Holder, and J. Stuart. The asymptotic optimal partition and extensions of the nonsubstitution theorem. Technical Report ??, Trinity University Mathematics, San Antonio, TX, 2001.

[14] A. Holder. Partitioning multiple objective solutions with applications in radiotherapy design. Technical Report 54, Trinity University Mathematics, 2001.

[15] R. Jeroslow. Asymptotic linear programming. Operations Research, 21:1128-1141, 1972.

[16] R. Jeroslow. Linear programs dependent on a single parameter. Discrete Mathematics, 6:119-140, 1973.

[17] K. Kuga. The non-substitution theorem: Multiple primary factors and the cost function approach. Technical Report Discussion Paper No. 529, The Institute of Social and Economic Research, Osaka Univeristy, Osaka, Japan, 2001.

[18] H. Kurz and N. Salvadori. Theory of Production: A long-period analysis. Cambridge University Press, New York, NY, 1995.

[19] J. Mirrlees. The dynamic nonsubstitution theorem. Review of Economic Studies, 36(105):67-76, 1969.

[20] C. Roos, T. Terlaky, and J.-Ph. Vial. Theory and Algorithms for Linear Optimization: An Interior Point Approach. John Wiley \& Sons, New York, NY, 1997.

[21] P. Samuelson. Abstract of a theorem concerning substitutability in open leontief models. In T. Koopmans, editor, Activity Analysis of Production and Allocation, page Chapter 7. Wiley, New York, NY, 1951.

[22] Adam Smith. The Wealth of Nations. Alfred A. Knopf, Inc., New York, 1991. 
[23] H. Ying. A canonical form for pencils of matrices with applications to asymptotic linear programs. Linear Algebra and its Applications, 234:97123, 1996. 